\title{
TRANSLATIONAL ADDITION THEOREMS FOR SPHERICAL VECTOR WAVE FUNCTIONS*
}

\author{
BY \\ ORVAL R. CRUZAN \\ Diamond Ordnance Fuze Laboratories \\ Washington, D. C.
}

\begin{abstract}
Translational addition theorems for spherical vector wave functions have been derived in a reduced form. The reduction has been accomplished by the use of formulas relating the coefficients that arise in the expansion of the product of two associated Legendre functions. These addition theorems should be useful, in general, in those cases involving electromagnetic sources and spherical bodies.

1. Introduction. When electromagnetic waves interact with spherical bodies, it becomes desirable in many problems to expand the fields in terms of spherical vector wave functions. Furthermore, it is helpful to have addition theorems for the wave functions, e.g., in the case of two or more spherical bodies. Here we need to expand the secondary electromagnetic field of each sphere about the other in order to readily apply the boundary conditions appropriate to each spherical body.

Such addition theorems have been obtained by Stein [1]. As given for the case of coordinate translation they are not in the most desirable form, in that the coefficients involved consist of several terms each. Although these coefficients were not reduced, it was stated that certain recursion formulas might be useful [2].

It turns out that in addition to the two recursion formulas, Eqs. (A-7) and (A-8), that Stein [3] considered particularly useful, four other formulas are required, two of which are of the recursion form; the other two have a quasi-recurrent form, as shown by Eqs. (A-3) and (A-4). These additional formulas have been obtained, and the coefficients reduced to one term each [4]. Moreover, the derivation of the addition theorems has been made in a straightforward and rigorous way, by making use of the orthogonal properties of the angular functions and of the vector wave functions.

Besides the formulas sufficient for the reduction of the addition theorems, several three term recursion formulas have been derived. These should be helpful for computing the values of the coefficients in the expansion of the product of two associated Legendre functions.
\end{abstract}

2. Spherical vector wave functions [5]. Divergenceless solutions of the vector Helmholtz equation,

$$
\nabla \nabla \cdot \mathbf{c}-\nabla \times \nabla \times \mathbf{c}+k^{2} \mathbf{c}=0
$$

where $k$ is the propagation constant, are the two vector wave functions $\mathbf{M}$ and $\mathbf{N}$. The relations between these two are

$$
\begin{aligned}
k \mathbf{N} & =\nabla \times \mathbf{M} \\
k \mathbf{M} & =\nabla \times \mathbf{N} .
\end{aligned}
$$


In spherical coordinates, $\mathbf{M}$ is given by the formula

$$
\mathbf{M}=\nabla \times \mathbf{r} \psi,
$$

where $\mathbf{r}$ is a radial vector, and $\psi$ is a solution of the scalar Helmholtz equation,

$$
\nabla^{2} \psi+k^{2} \psi=0 \text {. }
$$

By means of vector identities, Eq. (3) can be put in the alternative form,

$$
\mathbf{M}=\nabla \psi \times \mathbf{r},
$$

since $\nabla \times \mathbf{r}=0$. Omitting the time factor exp $(-i \omega t)$, the explicit expressions for $\mathbf{M}$ and $\mathbf{N}$, respectively, are [1]:

$$
\mathbf{m}_{m n}=\frac{i m}{\sin \theta} z_{n}(k r) P_{n}^{m}(\cos \theta) \exp (i m \phi) \mathbf{i}_{\theta}-z_{n}(k r) \frac{\partial P_{n}^{m}}{\partial \theta}(\cos \theta) \exp (i m \phi) \mathbf{i}_{\phi}
$$

and

$\mathbf{n}_{m n}=\frac{n(n+1)}{k r} z_{n}(k r) P_{n}^{m}(\cos \theta) \exp (i m \phi) \mathbf{i}_{r}+\frac{1}{k r} \frac{\partial}{\partial r}\left[r z_{n}(k r)\right] \frac{\partial P_{n}^{m}}{\partial \theta}\left(\cos _{-} \theta\right) \exp (i m \phi) \mathbf{i}_{\theta}$

$$
+\frac{i m}{k r \sin \theta} \frac{\partial}{\partial r}\left[r z_{n}(k r)\right] P_{n}^{m}(\cos \theta) \exp (i m \phi) \mathbf{i}_{\phi},
$$

where $z_{n}(k r)$ stands for any of the spherical radial functions.

3. Vector wave functions under coordinate translation [4]. Although in the following derivation of the translational addition theorems, waves convergent about the origin $0^{\prime}$ are used, the derivation is just as readily made for divergent waves. If the translation as illustrated in Fig. 1 is made, then

$$
\mathbf{r}=\mathbf{a}+\mathbf{r}^{\prime} .
$$

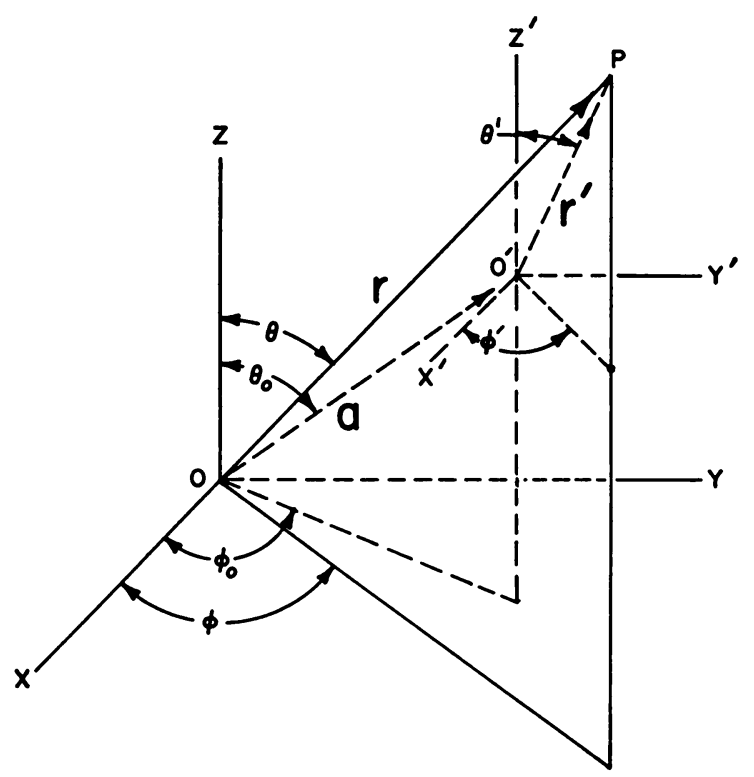

Fig. 1. Coordinate translation 
With this value of $\mathbf{r}$, we have, by using Eq. (5),

$$
\mathbf{M}=\nabla \psi \times \mathbf{a}+\nabla \psi \times \mathbf{r}^{\prime} .
$$

Since the gradient of a scalar quantity is invariant to a transformation of the coordinate system, then we may regard $\nabla \psi$ as being expressed in terms of the coordinates of the second system. As can be determined from Fig. 1,

$$
\mathbf{a}=a\left(\mathbf{i}_{x} \sin \theta_{0} \cos \phi_{0}+\mathbf{i}_{y} \sin \theta_{0} \sin \phi_{0}+\mathbf{i}_{z} \cos \theta_{0}\right) .
$$

Consequently, we have

$\mathbf{M}=a\left(\sin \theta_{0} \cos \phi_{0} \nabla \psi \times \mathbf{i}_{x}+\sin \theta_{0} \sin \phi_{0} \nabla \psi \times \mathbf{i}_{y}+\cos \theta_{0} \nabla \psi \times \mathbf{i}_{z}\right)$

$$
+\nabla \psi \times \mathbf{r}^{\prime} \text {. }
$$

The unit vectors $\left(\mathbf{i}_{x}, \mathbf{i}_{y}, \mathbf{i}_{z}\right)$ in terms of the unit vectors in the second coordinate system are

$$
\begin{aligned}
& \mathbf{i}_{x}=\mathbf{i}_{r^{\prime}} \sin \theta^{\prime} \cos \phi^{\prime}+\mathbf{i}_{\theta^{\prime}}, \cos \theta^{\prime} \cos \phi^{\prime}-\mathbf{i}_{\phi^{\prime}} \sin \phi^{\prime}, \\
& \mathbf{i}_{y}=\mathbf{i}_{r^{\prime}} \sin \theta^{\prime} \sin \phi^{\prime}+\mathbf{i}_{\theta^{\prime}} \cos \theta^{\prime} \sin \phi^{\prime}+\mathbf{i}_{\phi^{\prime}} \cos \phi^{\prime}, \\
& \mathbf{i}_{z}=\mathbf{i}_{r^{\prime}} \cos \theta^{\prime}-\mathbf{i}_{\theta^{\prime}} \sin \theta^{\prime} .
\end{aligned}
$$

If in Eq. $(B-1)$ we put

$$
\begin{aligned}
A(\mu, \nu)=(-1)^{\mu} i^{\nu-n}(2 \nu+1) & \\
& \cdot \sum_{\nu} i^{p} a(m, n|-\mu, \nu| p) z_{p}(k a) P_{p}^{m-\mu}\left(\cos \theta_{0}\right) \exp \left[i(m-\mu) \phi_{0}\right],
\end{aligned}
$$

where the $a(m, n|-\mu, \nu| p)$ 's are given in appendix $\mathrm{A}$, then

$$
\psi=\sum_{\nu=0}^{\infty} \sum_{\mu=-\nu}^{\nu} A(\mu, \nu) j_{\nu}\left(k r^{\prime}\right) P_{\nu}^{\mu}\left(\cos \theta^{\prime}\right) \exp \left(i \mu \phi^{\prime}\right) .
$$

Using this expression for $\psi$, we obtain, in view of Eq. (5), for the last term of the right member of Eq. (10),

$$
\nabla \psi \times \mathbf{r}^{\prime}=\sum_{\nu=0}^{\infty} \sum_{\mu=-\nu}^{\nu} A(\mu, \nu) \mathbf{m}_{\mu \nu}^{\prime},
$$

where $\mathrm{m}_{\mu \nu}^{\prime}$ is of the same form as $\mathrm{m}_{n m}$, Eqs. (6), but is referred to the second coordinate system. By using the orthogonal properties of the angular functions and of the vector wave functions, it can be shown that the other vector quantities in Eq. (10) are

$$
\begin{aligned}
& \nabla \psi \times \mathbf{i}_{x}=\sum_{\nu=0}^{\infty} \sum_{\mu=-\nu}^{\theta}\left(a_{\mu \nu}^{\prime} \mathbf{m}_{\mu \nu}^{\prime}+b_{\mu \nu}^{\prime} \mathbf{n}_{\mu \nu}^{\prime}\right), \\
& \nabla \psi \times \mathbf{i}_{\nu}=\sum_{\nu=0}^{\infty} \sum_{\mu=-\nu}^{\infty}\left(a_{\mu \nu}^{\prime \prime} \mathbf{m}_{\mu \nu}^{\prime}+b_{\mu \nu}^{\prime \prime} \mathbf{n}_{\mu \nu}^{\prime}\right), \\
& \nabla \psi \times \mathbf{i}_{z}=\sum_{\nu=0}^{\infty} \sum_{\mu=-\nu}^{\infty}\left(a_{\mu \nu}^{\prime \prime \prime} \mathbf{m}_{\mu \nu}^{\prime}+b_{\mu \nu}^{\prime \prime \prime} \mathbf{n}_{\mu \nu}^{\prime}\right) .
\end{aligned}
$$


where

$$
\begin{aligned}
a_{\mu \nu}^{\prime} & =\frac{\nu}{2 \nu(\nu+1)}\left\{\frac{\nu+1}{2 \nu-1}[A(\mu-1, \nu-1)-(\nu-\mu)(\nu-\mu-1) A(\mu+1, \nu-1)]\right. \\
& \left.+\frac{\nu}{2 \nu+3}[(\nu+\mu+2)(\nu+\mu+1) A(\mu+1, \nu+1)-A(\mu-1, \nu+1)]\right\}, \\
a_{\mu \nu}^{\prime \prime} & =\frac{-i k}{2 \nu(\nu+1)}\left\{\frac{\nu+1}{2 \nu-1}[A(\mu-1, \nu-1)+(\nu-\mu)(\nu-\mu-1) A(\mu+1, \nu-1)]\right. \\
& \left.-\frac{\nu}{2 \nu+3}[(\nu+\mu+2)(\nu+\mu+1) A(\mu+1, \nu+1)+A(\mu-1, \nu+1)]\right\}, \\
& a_{\mu \nu}^{\prime \prime \prime}=\frac{k}{\nu(\nu+1)}\left\{\frac{(\nu+1)(\nu-\mu)}{2 \nu-1} A(\mu, \nu-1)+\frac{\nu(\nu+\mu+1)}{2 \nu+3} A(\mu, \nu+1)\right\},
\end{aligned}
$$

and

$$
\begin{aligned}
& b_{\mu \nu}^{\prime}=\frac{-i k}{2 \nu(\nu+1)}[(\nu-\mu)(\nu+\mu+1) A(\mu+1, \nu)+A(\mu-1, \nu)], \\
& b_{\mu \nu}^{\prime \prime}=\frac{k}{2 \nu(\nu+1)}[(\nu-\mu)(\nu+\mu+1) A(\mu+1, \nu)-A(\mu-1, \nu)], \\
& b_{\mu \nu}^{\prime \prime \prime}=\frac{i k \mu}{\nu(\nu+1)} A(\mu, \nu),
\end{aligned}
$$

and $\mathbf{n}_{\mu \nu}^{\prime}$, is of the same form as $\mathbf{n}_{m n}$, Eqs. (6), except that it is referred to the second coordinate system.

In reducing Eq. (10), we may, by using Eqs. (15), set

$$
A_{\mu \nu}=\sin \theta_{0} \cos \phi_{0} a_{\mu \nu}^{\prime}+\sin \theta_{0} \sin \phi_{0} a_{\mu \nu}^{\prime \prime}+\cos \theta_{0} a_{\mu \nu}^{\prime \prime \prime} .
$$

If we denote the coefficient of the vector wave function $\mathbf{m}_{\mu \nu}^{\prime}$ by $A_{\mu \nu}^{m n}$, then from Eqs. (14), (15), and (18), we obtain

$$
A_{\mu \nu}^{m n}=a A_{\mu \nu}+A(\mu, \nu) .
$$

If we use the recursion formulas, Eqs. (A-6) through (A-9), and the appropriate relations between the spherical radial functions, then we have

$A_{\mu \nu}^{m n}=(-1)^{\mu} \sum_{p} a(m, n|-\mu, \nu| p) a(n, \nu, p) z_{p}(k a) P_{p}^{m-\mu}\left(\cos \theta_{0}\right) \exp \left[i(m-\mu) \phi_{0}\right]$,

where

$$
\begin{aligned}
a(n, \nu, p)=i^{\nu+\nu-n}[2 \nu(\nu+1) & (2 \nu+1)+(\nu+1)(n-\nu+p+1)(n+\nu-p) \\
& -\nu(\nu-n+p+1)(n+\nu+p+2)] /[2 \nu(\nu+1)] .
\end{aligned}
$$

For the coefficient of $\mathbf{n}_{\mu \nu}^{\prime}$, we may set

$$
B_{\mu \nu}^{m n}=a\left(\sin \theta_{0} \cos \phi_{0} b_{\mu \nu}^{\prime}+\sin \theta_{0} \sin \phi_{0} b_{\mu \nu}^{\prime \prime}+\cos \theta_{0} b_{\mu \nu}^{\prime \prime \prime}\right) .
$$

Using Eqs. (12), (17), (A-10), and (A-3) and the appropriate relations for the spherical radial functions, we obtain

$$
\begin{aligned}
B_{\mu \nu}^{m n}=(-1)^{\mu+1} \sum_{\nu} a(m, n|-\mu, \nu| p, p-1) b(n, \nu, p) z_{p}(k a) P_{p}^{m-\mu}\left(\cos \theta_{0}\right) & \cdot \exp \left[i(m-\mu) \phi_{0}\right],
\end{aligned}
$$


where

$$
\begin{array}{r}
b(n, \nu, p)=i^{\nu+p-n}[(n+\nu+p+1)(\nu-n+p)(n-\nu+p)(n+\nu-p+1)]^{1 / 2} \\
\cdot(2 \nu+1) /[2 \nu(\nu+1)] .
\end{array}
$$

4. Addition theorems. In summary, we have the translational addition theorems for spherical vector wave functions

Theorem I:

$$
\mathbf{m}_{m n}=\sum_{\nu=0}^{\infty} \sum_{\mu=-\nu}^{\nu}\left(A_{\mu \nu}^{m n} \mathbf{m}_{\mu \nu}^{\prime}+B_{\mu \nu}^{m n} \mathbf{n}_{\mu \nu}^{\prime}\right),
$$

Theorem II: $\quad \mathbf{n}_{m n}=\sum_{\nu=0}^{\infty} \sum_{\mu=-\nu}^{\nu}\left(A_{\mu \nu}^{m n} n_{\mu \nu}^{\prime}+B_{\mu \nu}^{m n} \mathbf{m}_{\mu \nu}^{\prime}\right)$,

where

$$
\begin{gathered}
A_{\mu \nu}^{m n}=(-1)^{\mu} \sum_{\nu} a(m, n|-\mu, \nu| p) a(n, \nu, p) z_{p}(k a) P_{p}^{m-\mu}\left(\cos \theta_{0}\right) \exp \left[i(m-\mu) \phi_{0}\right], \\
B_{\mu \nu}^{m n}=(-1)^{\mu+1} \sum_{p} a(m, n|-\mu, \nu| p, p-1) b(n, \nu, p) z_{p}(k a) P_{p}^{m-\mu}\left(\cos \theta_{0}\right) \exp \left[i(m-\mu) \phi_{0}\right], \\
\begin{array}{r}
a(n, \nu, p)=i^{\nu+p-n}[2 \nu(\nu+1)(2 \nu+1)+(\nu+1)(n-\nu+p+1)(n+\nu-p) \\
-\nu(\nu-n+p+1)(n+\nu+p+2)] /[2 \nu(\nu+1)],
\end{array}
\end{gathered}
$$

and

$$
\begin{array}{r}
b(n, \nu, p)=i^{\nu+p-n}[(n+\nu+p+1)(\nu-n+p)(n-\nu+p)(n+\nu-p+1)]^{1 / 2} \\
\cdot(2 \nu+1) /[2 \nu(\nu+1)] .
\end{array}
$$

\section{APPENDIX A}

Relationships involving the coefficients in the expansion of $P_{n}^{m} P_{\nu}^{\mu}$. In the derivation of the addition theorem for the spherical scalar wave function (appendix $B$ ) there occurs the product $P_{n}^{m} P^{\mu}$ of two associated Legendre functions in which the argument is $\eta=\cos \theta$. This product can be expressed in terms of the expansion [2, 4],

$$
P_{n}^{m} P_{\nu}^{\mu}=\sum_{p} a(m, n|\mu, \nu| p) P_{p}^{m+\mu}
$$

where

$$
\begin{aligned}
& a(m, n|\mu, \nu| p)=(-1)^{m+\mu}(2 p+1)\left[\frac{(n+m) !(\nu+\mu) !(p-m-\mu) !}{(n-m) !(\nu-\mu) !(p+m+\mu) !}\right]^{1 / 2} \\
& \cdot\left[\begin{array}{lll}
n & \nu & p \\
0 & 0 & 0
\end{array}\right]\left[\begin{array}{lll}
n & \nu & p \\
m & \mu & -m-\mu
\end{array}\right) \text {. }
\end{aligned}
$$

and

$$
\left[\begin{array}{lll}
j_{1} & j_{2} & j_{3} \\
m_{1} & m_{2} & m_{3}
\end{array}\right)
$$

is the Wigner $3-j$ symbol [6]. 
For the reduction of the coefficients involved in the addition theorems, it is necessary to obtain formulas simplifying various groupings of the $a(m, n|\mu, \nu| p)$ 's. From the formulas connecting the Wigner $3-j$ and $6-j$ symbols [6], and the formula for the $a(m, n|-\mu, \nu| p)$, Eq. (A-2), we can obtain the following relationships [4] $(2 p-1)[(n+\nu+p+1)(\nu-n+p)(n-\nu+p)(n+\nu-p+1)]^{1 / 2}$

$$
\begin{aligned}
& \cdot a(m, n|\mu, \nu| p, p-1) \\
= & (2 p+1)[(\nu+\mu)(\nu-\mu+1) a(m, n|\mu-1, \nu| p-1) \\
& -(p-m-\mu)(p-m-\mu-1) a(m, n|\mu+1, \nu| p-1) \\
& -2 \mu(p-m-\mu) a(m, n|\mu, \nu| p-1) 1 .
\end{aligned}
$$

$(2 p+3)[(n+\nu-p)(n-\nu+p+1)(\nu+p-n+1)(n+\nu+p+2)]^{1 / 2}$

$$
\begin{aligned}
& \cdot a(m, n|\mu, \nu| p, p+1) \\
= & (2 p+1)[(p+m+\mu+1)(p+m+\mu+2) a(m, n|\mu+1, \nu| p+1) \\
& -(\nu+\mu)(\nu-\mu+1) a(m, n|\mu-1, \nu| p+1) \\
& -2 \mu(p+m+\mu+1) a(m, n|\mu, \nu| p+1)],
\end{aligned}
$$

where

$$
\begin{aligned}
& a(m, n|\mu, \nu| p, q)=(-1)^{m+\mu}\left[\frac{(n+m) !(\nu+\mu) !(p-\mu-m) !}{(n-m) !(\nu-\mu) !(p+\mu+m) !}\right]^{1 / 2}(2 p+1) \\
& \cdot\left[\begin{array}{lll}
n & \nu & p \\
m & \mu & -m-\mu
\end{array}\right]\left[\begin{array}{lll}
n & \nu & q \\
0 & 0 & 0
\end{array}\right] \text {. }
\end{aligned}
$$

$$
\begin{aligned}
(2 p-1)(n+\nu- & p)(n-\nu+p+1) a(m, n|\mu, \nu-1| p) \\
= & (2 p+1)[2(\nu-\mu)(p-m-\mu) a(m, n|\mu, \nu| p-1) \\
& -(\nu-\mu)(\nu-\mu+1) a(m, n|\mu-1, \nu| p-1) \\
& -(p-m-\mu-1)(p-m-\mu) a(m, n|\mu+1, \nu| p-1)]
\end{aligned}
$$

$(2 p+3)(n-\nu+p)(n+\nu-p+1) a(m, n|\mu, \nu+1| p)$

$$
\begin{aligned}
= & (2 p+1)[-(p+m+\mu+1)(p+m+\mu+2) a(m, n|\mu+1, \nu| p+1) \\
& +2(\nu+\mu+1)(p+m+\mu+1) a(m, n|\mu, \nu| p+1) \\
& -(\nu+\mu)(\nu+\mu+1) a(m, n|\mu-1, \nu| p+1)] .
\end{aligned}
$$

$(2 p-1)(n+\nu+p+2)(\nu-n+p+1) a(m, n|\mu, \nu+1| p)$

$$
\begin{aligned}
= & (2 p+1)[(p-m-\mu-1)(p-m-\mu) a(m, n|\mu+1, \nu| p-1) \\
& +2(\nu+\mu+1)(p-m-\mu) a(m, n|\mu, \nu| p-1) \\
& +(\nu+\mu)(\nu+\mu+1) a(m, n|\mu-1, \nu| p-1)] .
\end{aligned}
$$

$(2 p+3)(\nu-n+p)(n+\nu+p+1) a(m, n|\mu, \nu-1| p)$

$$
\begin{aligned}
= & (2 p+1)[(p+m+\mu+2)(p+m+\mu+1) a(m, n|\mu+1, \nu| p+1) \\
& +(\nu-\mu)(\nu-\mu+1) a(m, n|\mu-1, \nu| p+1) \\
& +2(\nu-\mu)(p+m+\mu+1) a(m, n|\mu, \nu| p+1)] .
\end{aligned}
$$


In addition to the above relationships for the $a(m, n|\mu, \nu| p)$ 's, there can be derived numerous recursion formulas based upon various recursion relations for the associated Legendre functions. By using the relationship for associated Legendre functions,

$$
\left(1-\eta^{2}\right)^{1 / 2}\left[P_{\nu}^{\mu+1}+(\nu+\mu)(\nu-\mu+1) P_{\nu}^{\mu-1}\right]-2 \mu \eta P_{\nu}^{\mu}=0,
$$

and multiplying by $P_{n}^{m}$, it can be shown that

$$
\begin{aligned}
(2 p-1) & {[(p+m+\mu+1)(p+m+\mu+2) a(m, n|\mu+1, \nu| p+1)} \\
& -(\nu+\mu)(\nu-\mu+1) a(m, n|\mu-1, \nu| p+1) \\
& -2 \mu(p+m+\mu+1) a(m, n|\mu, \nu| p+1)] \\
= & (2 p+3)[(p-m-\mu)(p-m-\mu-1) a(m, n|\mu+1, \nu| p-1) \\
& -(\nu+\mu)(\nu-\mu+1) a(m, n|\mu-1, \nu| p-1) \\
& +2 \mu(p-m-\mu) a(m, n|\mu, \nu| p-1)] .
\end{aligned}
$$

Certain three-term recursion formulas are also readily obtained. From the relation

$$
\frac{d}{d \theta}\left(P_{n}^{m} P_{v}^{\mu}\right)=P_{n}^{m} \frac{d P_{v}^{\mu}}{d \theta}+P_{v}^{\mu} \frac{d P_{n}^{m}}{d \theta},
$$

and the derivative formulas

and

$$
\frac{d P_{n^{\prime}}^{m \prime^{\prime}}}{d \theta}=\frac{m^{\prime} \eta}{\left(1-\eta^{2}\right)^{1 / 2}} P_{n^{\prime}}^{m^{\prime}}-P_{n^{\prime}}^{m^{\prime}+1}
$$

$$
\frac{d P_{n^{\prime}}^{m^{\prime}}}{d \theta}=\frac{-m^{\prime} \eta}{\left(1-\eta^{2}\right)^{1 / 2}} P_{n^{\prime}}^{m^{\prime}}+\left(n^{\prime}-m^{\prime}+1\right)\left(n^{\prime}+m^{\prime}\right) P_{n^{\prime}}^{m^{\prime}-1},
$$

one obtains respectively

$$
a(m, n|\mu, \nu| p)=a(m+1, n|\mu, \nu| p)+a(m, n|\mu+1, \nu| p)
$$

and

$$
\begin{aligned}
(p-m-\mu+1)(p+m+ & \mu) a(m, n|\mu, \nu| p) \\
= & (\nu+\mu)(\nu-\mu+1) a(m, n|\mu-1, \nu| p) \\
& +(n+m)(n-m+1) a(m-1, n|\mu, \nu| p) .
\end{aligned}
$$

From Eqs. (A-14) and (A-15) there can be obtained the formulas

$$
\begin{gathered}
{[(p+m+\mu)(p-m-\mu+1)+(\nu-\mu)(\nu+\mu+1)-(n+m)(n-m+1)]} \\
\cdot a(m, n|\mu, \nu| p)=(\nu+\mu)(\nu-\mu+1) a(m, n|\mu-1, \nu| p) \\
+(p-m-\mu)(p+m+\mu+1) a(m, n|\mu+1, \nu| p)
\end{gathered}
$$

and

$$
\begin{gathered}
{[(p+m+\mu)(p-m-\mu+1)+(n-m)(n+m+1)-(\nu+\mu)(\nu-\mu+1)]} \\
\cdot a(m, n|\mu, \nu| p)=(n+m)(n-m+1) a(m-1, n|\mu, \nu| p) \\
+(p-m-\mu)(p+m+\mu+1) a(m+1, n|\mu, \nu| p) .
\end{gathered}
$$




\section{APPENDIX B}

\section{Addition theorems for spherical scalar wave functions}

In this appendix, the addition theorems for the scalar wave functions will be listed, with appropriate limits, since they have been already derived [7]. The addition theorems given here are for the case of coordinate translation illustrated in Fig. 1. The more correct forms for the theorems [1] are

$$
\begin{aligned}
z_{n}(k r) P_{n}^{m}(\cos \theta) \exp (i m \phi)=\sum_{\nu=0}^{\infty} \sum_{\mu=-\nu} \sum_{\nu}(-1)^{\mu} i^{\nu+p-n}(2 \nu+1) a(m, n|-\mu, \nu| p) \\
\cdot j_{\nu}\left(k r^{\prime}\right) z_{p}(k a) P_{\nu}^{\mu}\left(\cos \theta^{\prime}\right) P_{p}^{m-\mu}\left(\cos \theta_{0}\right) \exp \left[i(m-\mu) \phi_{0}\right] \exp \left(i \mu \phi^{\prime}\right) \quad r^{\prime} \leq a \\
=\sum_{\nu=0}^{\infty} \sum_{\mu=-\nu} \sum_{p}(-1)^{\mu} i^{\nu+p-n}(2 \nu+1) a(m, n|-\mu, \nu| p) \\
\cdot j_{\nu}(k a) z_{p}\left(k r^{\prime}\right) P_{\nu}^{\mu}\left(\cos \theta_{0}\right) P_{p}^{m-\mu}\left(\cos \theta^{\prime}\right) \exp \left[i(m-\mu) \phi^{\prime}\right] \exp \left(i \mu \phi_{0}\right) \quad r^{\prime} \geq a .
\end{aligned}
$$

The symbol $z_{n}(k r)$ stands for the spherical Bessel, Neumann, or Hankel (first or second kind) function. It can be shown that either Eq. (B-1) or Eq. (B-2) may be used with $z_{n}(k r)=j_{n}(k r)$, without restriction on the relative size of $r^{\prime}$ and $a$.

\section{REFERENCES}

1. S. Stein, Addition theorems for spherical wave functions, Quart. Appl. Math. 19, 15-24 (1961)

2. S. Stein, Addition theorems for spherical wave functions, Hermes Electronics Co., Cambridge, Mass., Tech. Rept. No. 1 under Nonr-2632(00), Hermes Electronics Rept. M-697, 15 August 1959

3. S. Stein, Ibid., p. 33

4. O. R. Cruzan, Translational addition theorems for spherical vector wave functions, Diamond Ordnance Fuze Labs., Washington 25, D. C., Tech. Rept. TR-906, 10 March 1961

5. J. A. Stratton, Electromagnetic theory, McGraw-Hill Book Co., Inc., New York, N. Y., 1941, pp. $392-423$

6. A. R. Edmonds, Angular momentum in quantum mechanics, Princeton Univ. Press, Princeton, N. J., 1957

7. B. Friedman and J. Russek, Addition theorems for spherical waves, Quart. Appl. Math. 12, 13-23 (1954) 